\title{
Synthesis and Characterizations of Bare CdS Nanocrystals Using Chemical Precipitation Method for Photoluminescence Application
}

\author{
H. L. Lee, ${ }^{1}$ A. M. Issam, ${ }^{2}$ M. Belmahi, ${ }^{3}$ M. B. Assouar, ${ }^{3}$ H. Rinnert, ${ }^{3}$ and M. Alnot ${ }^{3}$ \\ ${ }^{1}$ Nanoscience Research Laboratory, School of Chemical Sciences, University Sains Malaysia, 11800 Penang, Malaysia \\ ${ }^{2}$ School of Industrial Technology, University Sains Malaysia, 11800 Penang, Malaysia \\ ${ }^{3}$ Institut Jean Lamour, UMR 7198 CNRS, Faculté des Sciences et Techniques, Nancy Université, Université P. Verlaine, B.P. 239,54506 \\ Vandoeuvre les Nancy Cedex, France \\ Correspondence should be addressed to H. L. Lee, hooiling78@gmail.com
}

Received 15 June 2009; Revised 10 August 2009; Accepted 4 September 2009

Recommended by Sherine Obare

\begin{abstract}
Bare cadmium sulfide (CdS) nanocrystals were successfully synthesized by the thermolysis of a single-source organometallic precursor, cadmium chloride hemipentahydrate $\left(\mathrm{CdCl}_{2} \cdot 2.5 \mathrm{H}_{2} \mathrm{O}\right)$ with thiourea in ethanol. The microstructure of the CdS samples was characterized using XRD, TEM, and Raman spectroscopy. The XRD's results showed that there was a transformation from cubic to hexagonal crystalline phase when higher mass of $\mathrm{CdCl}_{2} \cdot 2.5 \mathrm{H}_{2} \mathrm{O}$ was used. Further experimental with different $\mathrm{Cd}^{2+}$ source showed ion $\mathrm{Cl}^{-}$originated from $\mathrm{CdCl}_{2} \cdot 2.5 \mathrm{H}_{2} \mathrm{O}$ attributed to this crystalline phase transformation. The UV-Visible analysis indicated that quantum confinement effect took place when compared to the bulk CdS. However, the photoluminescence experiments revealed that the red-light emission was observed in all samples. This finding could be ascribed to deep trap defects that were due to sulfur vacancies as suggested by XPS and also the fact that the bare CdS nanoparticles are in contact with each other as shown in the TEM images.
\end{abstract}

Copyright ( $) 2009$ H. L. Lee et al. This is an open access article distributed under the Creative Commons Attribution License, which permits unrestricted use, distribution, and reproduction in any medium, provided the original work is properly cited.

\section{Introduction}

Semiconductor quantum dots (QDs) have been extensively researched due to their unique size-dependent optical and electrical properties $[1,2]$. These unique properties show many potential applications in optoelectronic devices [3]. Group II-VI semiconductor nanocrystals such as CdS, CdSe, and CdTe have been investigated widely as they emit in the visible range [4].

Cadmium sulfide (CdS) is a direct band gap semiconductor with $\mathrm{Eg} \sim 2.5 \mathrm{eV}$ [5]. It is used in photodetectors, optoelectronics, and for solar cell applications [5]. A number of sources for sulfur for preparation of CdS nanoparticles have been reported. Sodium sulphide [6], hydrogen sulphide gas [7], and thiourea [8] are the most commonly used for solution-based preparations of nanosized CdS. In view of that, remarkable efforts have been made to develop methods for high-quality CdS nanocrystals such as controlled precipitation reactions, the thermolysis of single precursors at relatively high temperature, and rapid hot-injection-based synthesis. The rapid hot-injection-based synthesis gives the effective separation of the nucleation and growth stages. Various preparation methods to control the size of semiconductor nanocrystallites have been developed: (a) using stabilizers [6] and polymers $[9,10]$, (b) size fractionation by exclusion chromatography [11], and (c) size selective chromatography.

Photoluminescence (PL) spectra for CdS nanocrystals consist of green, yellow, and red band emission [12]. The green and yellow bands are associated with the band-edge emissions whereas the low-energy band or red band is related to surface defects or surface states $[12,13]$. Nevertheless, the red band usually dictates the PL spectra when the nanocrystals are not molecular capped (bare) [12]. In the case when the broad band is in the range of $700-800 \mathrm{~nm}$, 
it could be attributed to the complex defects including the cadmium vacancy [14] or sulfur vacancy.

Generally, CdS shows dimorphism of cubic form (zinc blend type) and hexagonal form (wurtzite type) [15]. The crystalline structure of semiconductor nanocrystallite will determine the optical properties. The difference in crystalline phase will lead to considerable change in the effective masses of electrons and holes in their electronic bands. Hexagonal phase of CdS is thermodynamically-controlled (stable) as compared with the cubic phase of CdS which is kinetically-controlled (metastable) [16]. Hence, researchers would favour a reaction that forms thermodynamically stable nanoscale CdS materials. Our task is to study the effect of $\mathrm{Cd}^{2+}$ ions mass concentration and source of $\mathrm{Cd}^{2+}$ ions in determining the crystalline phase of the bare CdS nanoparticles. In this study, we employed a facile, low-cost, and environmentally friendly synthetic method which is based on thermolysis of a single-source organometallic precursor. This method is adapted with slight modification from Tong and Zhu [8]. We also have performed several instrumentation techniques such as XRD, SEM-EDX, UV-Vis Spectroscopy, TEM, XPS, and PL spectroscopy to characterize the bare CdS nanoparticles.

\section{Experimental}

2.1. Materials. The starting materials for the synthesis of CdS were $\mathrm{CdCl}_{2} \cdot 2.5 \mathrm{H}_{2} \mathrm{O}$, thiourea, absolute ethanol, and sodium hydroxide that were purchased and used as received. All the reagents were of analytical purity. Doubly distilled water was used throughout the experiment which was conducted under air atmosphere.

2.2. Synthesis. Solution A was prepared by dissolving $0.11 \mathrm{mmol}(24 \mathrm{mg})$ of cadmium chloride hemipentahydrate $\left(\mathrm{CdCl}_{2} .2 .5 \mathrm{H}_{2} \mathrm{O}\right)$ and $0.63 \mathrm{mmol}(48 \mathrm{mg})$ of thiourea $\left(\left(\mathrm{NH}_{2}\right)_{2} \mathrm{CS}\right)$ into $30 \mathrm{~mL}$ of ethanol in a flask under magnetic stirring at $160^{\circ} \mathrm{C}$ in the silicon oil bath. Solution B was prepared by dissolving $0.25 \mathrm{mmol}(10 \mathrm{mg})$ of sodium hydroxide $(\mathrm{NaOH})$ in $10 \mathrm{~mL}$ ethanol. Then, $10 \mathrm{~mL}$ of solution $\mathrm{B}$ was rapidly pipetted into the solution. The mixed solution was held under reflux at $160^{\circ} \mathrm{C}$ for 10 hours. A white precursor was formed upon mixing of Solution B into Solution A. The yellow precipitates was collected by centrifugation and washed repeatedly with doubly distilled water before being dried in the vacuum oven at $50^{\circ} \mathrm{C}$ for 24 hours. The product was then used in the characterization work.

Similar approach with cadmium acetate (CdOAc) is employed as to study the effect of the cadmium source in influencing the crystalline phase of the synthesized CdS.

2.3. Characterization. All samples were characterized by powder X-ray Diffraction (XRD). Data were collected on a SIEMENS D5000 X-ray diffractometer with monochromatic $\mathrm{Cu}-\mathrm{K} \alpha(\lambda=1.5405 \AA)$ radiation filter in the $2 \theta$ range $0-100^{\circ}$. U-2000 Hitachi UV-visible spectrophotometer was used to record the UV-visible absorption spectrum of the as-prepared particles. Average particle size of the particles and selected area electron diffraction (SAED) analysis was obtained by using transmission electron microscope (TEM) model Philips CM20 operating at $200 \mathrm{kV}$. One mg of sample was dispersed in $3 \mathrm{~mL}$ of doubly distilled water and ultrasonicated for 5 minutes. A few drops of dispersion were placed on a carbon coated copper grid and the solvent was evaporated off. An Electron Energy Dispersive (EDX) model Leica Cambridge S360 was used to observe the morphology and composition of the product. The powders were mounted onto the metal holder through a double-sided tape and then coated with gold. The particle size of the sample was counted using AnalySIS Docu Image Analyzer Ver. 3.1, GmbH. Micro-Raman Spectroscopy (Jobin Yvon Horiba UV-VIS NIR micro-Raman) is performed at room temperature, by using a nonpolarized $\mathrm{Ar}^{+}$laser $(514.9 \mathrm{~nm})$ in an almost back-scattering geometry. Low-power beams, typically $20 \mathrm{~mW}$ at the sample surface, were used to avoid heating and any apparent damage to the samples during the analysis.

For the photoluminescence (PL) experiment the excitation was obtained by the UV lines from a $200 \mathrm{~W}$ mercury arc lamp source (Oriel, model $200 \mathrm{~W}$ ). Optical emission was analyzed by a monochromator (Triax 190, Jobin Yvon) equipped with a 150 grooves $/ \mathrm{mm}$ grating and by a CCD camera (Jobin Yvon) cooled at $140 \mathrm{~K}$. The acquisition time was around 1 second. The response of the detection system was precisely calibrated with a tungsten wire calibration source. A cryostat was used to perform the measurements at $77 \mathrm{~K}$. The chemical composition of the samples was characterised after argon bombardment by X-ray photoemission spectroscopy (XPS, Mg K $\alpha: h v=1253.6 \mathrm{eV}$ ) from ESCALAB MK II VG. Spectrometer energy calibration was done with the $\mathrm{Au}_{4} \mathrm{f}_{7 / 2}$ photoelectron level using a gold foil. Due to surface electrostatic charges, which results in retarding effects, the corresponding energy scales of the XPS spectra were normalized using the fixed energy position of the $\mathrm{C} 1 \mathrm{~s}$ photoelectron line of the carbon contamination. The real value of C1s is between 284 [17] and $285 \mathrm{eV} \mathrm{[18].}$ Nevertheless, most authors take $285 \mathrm{eV}$ as the reference for C1s. Therefore, the value of $284 \mathrm{eV}$ from Tong et al.[17] is shifted by $1 \mathrm{eV}$ to $285 \mathrm{eV}$ to have the same energy for C1s with other reported journal [18]. Hence, the level in this paper is $1 \mathrm{eV}$ higher in binding energy.

\section{Results and Discussion}

3.1. Characterization. A survey of the samples shows that before the reaction is conducted, the original solution of cadmium chloride and thiourea is colourless. Upon the addition of sodium hydroxide, we observe that white precursors are formed. According to Tong and Zhu [8], this is due to the formation of cadmium hydroxide. After 5 minutes, the solution gradually changes to green-yellow and finally to bright yellow precipitates. The reaction of cadmium hydroxide and thiourea produces the side products of diazomethane $\left(\mathrm{CH}_{2} \mathrm{~N}_{2}\right)$, water, $\mathrm{Cd}^{2+}$ ions and $\mathrm{S}^{2-}$ ions. These ions eventually form as $\mathrm{CdS}$ nanoparticles that precipitate as yellow powders. The proposed reaction is indicated as below 
and agrees with the reported mechanism by Prabahar and Dhanam [19]:

$$
\begin{gathered}
\mathrm{CdCl}_{2} \cdot 2.5 \mathrm{H}_{2} \mathrm{O} \longrightarrow \mathrm{Cd}^{2+}+2 \mathrm{Cl}^{-}+2.5 \mathrm{H}_{2} \mathrm{O} \\
2 \mathrm{NaOH} \longrightarrow 2 \mathrm{Na}^{+}+2 \mathrm{OH}^{-} \\
\mathrm{Cd}^{2+}+2 \mathrm{OH}^{-} \longrightarrow \mathrm{Cd}(\mathrm{OH})_{2} \\
2 \mathrm{Na}^{+}+2 \mathrm{Cl}^{-} \longrightarrow 2 \mathrm{NaCl} \\
\left(\mathrm{NH}_{2}\right)_{2} \mathrm{CS}+\mathrm{Cd}(\mathrm{OH})_{2} \longrightarrow \mathrm{CH}_{2} \mathrm{~N}_{2}+2 \mathrm{H}_{2} \mathrm{O}+\mathrm{Cd}^{2+}+\mathrm{S}^{2-} \\
\mathrm{Cd}^{2+}+\mathrm{S}^{2-} \longrightarrow \mathrm{CdS}
\end{gathered}
$$$$
\text { Overall : } \mathrm{CdCl}_{2} \cdot 2.5 \mathrm{H}_{2} \mathrm{O}+\left(\mathrm{NH}_{2}\right)_{2} \mathrm{CS}+2 \mathrm{NaOH}
$$$$
\longrightarrow \mathrm{Cds}+\mathrm{CH}_{2} \mathrm{~N}_{2}+2 \mathrm{NaCl}+4.5 \mathrm{H}_{2} \mathrm{O}
$$

Temperature plays an important role in this method. Temperatures below $100^{\circ} \mathrm{C}$ would prolong the reaction time. After several attempts, we find that the temperature at $160^{\circ} \mathrm{C}$ is optimal for this reaction given that we employ a simple apparatus using a hot plate and silicon oil as the heating medium. The studies of the effect of cadmium concentration and source are then performed and characterizations with XRD, SEM-EDX, TEM, SAED, Raman Spectroscopy, UV-Vis spectroscopy, XPS and, PL spectrocopy analyses are carried out. These characterizations will be discussed in detail in the following sections.

\subsubsection{X-Ray Diffraction Technique (XRD).}

(i) Effect of cadmium concentration.

The influence of Effect of cadmium concentrations on the crystalline phase is studied.

$\mathrm{X}$-ray diffraction of the CdS prepared using cadmium chloride hemipetahydrate and thiourea at different composition ratios from $1: 2$ to $6: 1\left(\mathrm{CdCl}_{2}:\right.$ thiourea $)$ is shown in Figure 1. When CdS is prepared at the mass composition ratio of $\mathrm{CdCl}_{2}$ and thiourea at $1: 2$ and $1: 1$ (Figures 1(a) and 1(b)), three peaks are observed. These peaks are positioned with $2 \theta$ values of $26.6^{\circ}, 44.1^{\circ}$ and, $52.3^{\circ}$ which correspond to the Miller indices for the crystal plane of reflection of [111], [220], and [311]. The peaks are assignable to the pure face-centred cubic phase when compared with the standard reference (JCPDS 01080-0019). Similar observation is reported by Tong and Zhu [8] who synthesized CdS using cadmium chloride and thiourea however at the ratio of $1: 3$. Subsequently, when the mass composition of cadmium chloride to thiourea is increased to $2: 1$ (Figure $1(\mathrm{c})$ ), the peak at $48.1^{\circ}$ appears which is assignable to Miller indices for the [103] plance of reflection of from hexagonal crystalline phase. On the same account, peaks at $25.0^{\circ}, 26.5^{\circ}$, and $28.5^{\circ}$ have gradually appeared although the peaks are not separated clearly. This finding suggests the presence of cubic and hexagonal crystalline phase. When the mass of cadmium chloride is increased to $3: 1$ and higher (Figures $1(d)-1(f)$ ), a peak at

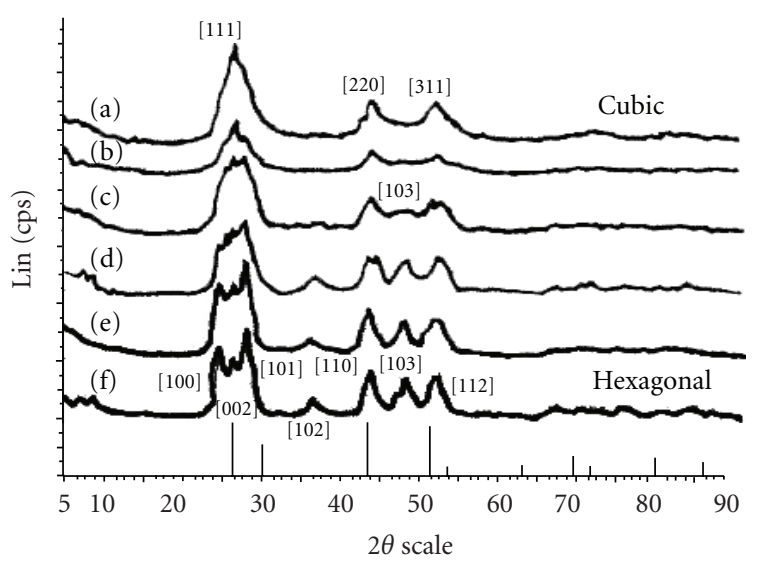

FIGURE 1: X-ray diffraction pattern of CdS nanoparticles in various ion $\mathrm{Cd}^{2+}$ from cadmium chloride to $\mathrm{S}^{2-}$ mass composition: (a) 1 : 2, (b) $1: 1$, (c) $2: 1$, (d) $3: 1$, (e) $4: 1$, and (f) $6: 1$.

$36.5^{\circ}$ emerges. It corresponds to the Miller indices for the crystalline plane of [102] in hexagonal phase. The other three peaks at $25.0^{\circ}, 26.5^{\circ}$, and $28.5^{\circ}$ could also be distinguished. Finally, when the mass composition of cadmium chloride is increased to 6 parts (Figure 1(f)), all the seven major peaks of hexagonal phase positioned at $24.8^{\circ}, 26.5^{\circ}, 28.2^{\circ}, 36.8^{\circ}$, $44.0^{\circ}, 48.2^{\circ}$, and $52.0^{\circ}$ are present in the sample (f). These peaks correspond to the Miller indices of [100], [002], [101], [102], [110], [103], and [112]. The diffractogram shows that a single hexagonal crystalline phase occurs according to the standard reference (JCPDS 01-080-0006). Compared with the bulk CdS, the diffraction peak of the nanoparticles is broadened due to the reduced size and the extensive surface defects [20].

\section{(ii) Effect of cadmium source.}

Subsequently, another source of $\mathrm{Cd}^{2+}$ is studied and compared with the previous experiment. Here, cadmium acetate $(\mathrm{CdOAc})$ is employed as the cadmium precursor whereas thiourea remains as the sulfur source. As depicted in Figure 2, the XRD shows that only reflection at $26.6^{\circ}$, $44.1^{\circ}$, and $52.3^{\circ}$ exists. The XRD pattern suggests the cubic CdS structures in Figures 2(a)-2(c) are synthesized from cadmium acetate. However, cubic structure of CdS vanishes gradually as the mass composition of cadmium acetate to thiourea is increased from 3 to 1 to 6 to 1 (Figure 2). No phase transformation from cubic to hexagonal is observed.

Based on the above results, it is possible to see that the crystalline phase can be controlled by manipulating the $\mathrm{CdCl}_{2}$ composition. In this study, the transformation of the crystalline phase from cubic to hexagonal is observed with increasing $\mathrm{CdCl}_{2}$ content. This phenomenon can be explained as being due to the change in atomic rearrangement upon the addition of $\mathrm{CdCl}_{2}$ in the $\mathrm{CdS}$ sample. $\mathrm{Cd}$ vacancies are created in the CdS matrix to maintain the overall charge neutrality by incorporating the $\mathrm{Cl}^{-}$ions [15]. The availability of space due to formation of $\mathrm{Cd}$ vacancies provides easy rearrangement of atoms. The excess 


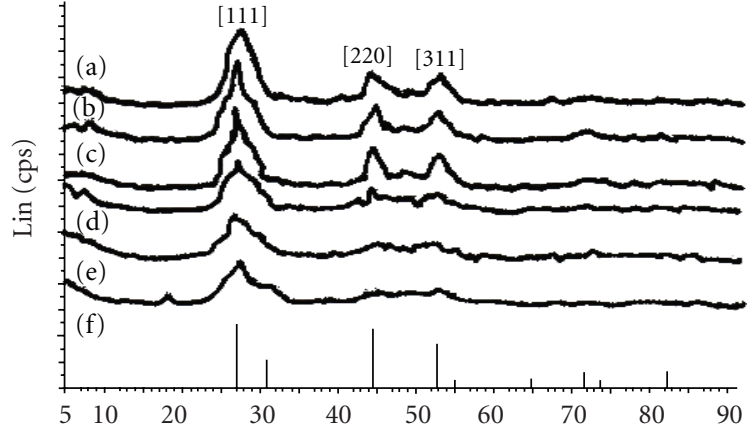

FIGURE 2: X-ray diffraction pattern of CdS nanoparticles in various ion $\mathrm{Cd}^{2+}$ from cadmium acetate to $\mathrm{S}^{2-}$ mass composition: (a) $1: 2$, (b) $1: 1$, (c) $2: 1$, (d) $3: 1$, (e) $4: 1$, and (f) $6: 1$.

of $\mathrm{CdCl}_{2}$ therefore indirectly modifies the stacking sequence of the face-centred cubic structure CdS to the more stable stacking structure of the hexagonal close-packed structure CdS. According to Matsumato and his coresearchers [21], the phase transformation from cubic to hexagonal in CdS needs the progressive movement of the cubic-hexagonal interface along the [111] direction of the cubic form which is also the [002] direction of the hexagonal form.

\subsubsection{Transmission Electron Microcopy (TEM) and Selected} Area Electron Diffraction (SAED). TEM images and the histogram of the size distribution of the prepared samples from cadmium chloride are shown in Figure 3. The particle sizes of more than 300 particles are calculated. The average particle size is between 4 and $6 \mathrm{~nm}$ for all the samples with more than $80 \%$ of them are at $5 \mathrm{~nm}$. The standard deviations are less than $1 \mathrm{~nm}$ in all samples.

The SAED images for three selected samples $(1: 2,3: 1$ and $6: 1$ ) are analysed. The SAED's images in Figures $3(\mathrm{~b})$ and 3(c) clearly show the hexagonal structure of CdS when the mass composition of $\mathrm{Cd}^{2+}: \mathrm{S}^{2-}$ is $3: 1$ and $6: 1$, respectively. The calculated $d$-spacings for all major rings in these samples correspond with the $d$-spacings from Pearson's Crystal data: 1120352 of CdS hexagonal crystalline phase. The results agree well with the Miller indices, of [100], [002], [101], [102], [110], [103], and [112] acquired from XRD analysis earlier (Figure 1). However, the SAED's image for sample in which the composition is $1: 2$ (Figure 3(a)) reveals that the three major rings could have formed [002], [110], and [200] lattices from hexagonal crystalline phase. On the other hand, the XRD result shows that cubic crystalline phase is formed. This anomalous result could be attributed to the overlapping between the XRD lines for hexagonal and cubic structures [5]. The overlapping is due to the Scherrer broadening that occurred because of the quantum confinement effect [20].

3.1.3. Scanning Electron Microscope-Energy Dispersive XRay (SEM-EDX). The synthesized samples from cadmium chloride are further characterized by SEM-EDX analysis for the evaluation of their composition and purity. The samples are observed to have near spherical particles and the overall morphology of the particles is almost homogenous (Figure 4). The EDX pattern for CdS powder is also obtained. It indicates the presence of $\mathrm{Cd}$ and $\mathrm{S}$. The atomic ratio of $\mathrm{Cd}$ $: S$ is $50.4: 49.6$ which can be translated into a stoichiometric ratio of approximately $1: 1$, agreeing with the formulation and formation of CdS.

3.1.4. Raman Spectroscopy. Raman scattering measurements are performed on the sample at the mass composition of $\mathrm{CdCl}_{2}$ : thiourea of $1: 2$ at $15^{\circ} \mathrm{C}$. It is well known that $\mathrm{CdS}$ is Raman active. The series of the CdS are confirmed with Raman spectroscopy as illustrated in Figure 5. Two characteristic CdS LO phonon peaks are observed, 1-LO at $296 \mathrm{~cm}^{-1}$ and its overtone $2-\mathrm{LO}$ at $591 \mathrm{~cm}^{-1}$ [22]. The result is in agreement with the report by Scott and Daman [22] and other researchers [23]. They have observed the surface mode peak of small CdS crystallites of size $=1 \mu \mathrm{m}$ at 296 $\mathrm{cm}^{-l}$ and its second-order peak at $592 \mathrm{~cm}^{-1}$. Raman peaks do not shift significantly upon the transformation to the hexagonal phase. This is because the frequency of LO mode in cubic phase is nearly the same as that of $A_{1}(L O)$ in the hexagonal phase [24]. The relative intensities of the 1$\mathrm{LO}$ and 2-LO lines change with the transformation of the crystalline phase from face-centred cubic to hexagonal in the current study. $\mathrm{I}_{2} \mathrm{LO} / \mathrm{I}_{1} \mathrm{LO}$ is a measure of the strength of the electron-phonon interaction [25]. We observe the difference in the symmetry of LO phonons in the hexagonal phase with respect to that of the LO phonon in the cubic phase could be one of the reasons for substantially higher $\mathrm{I}_{2} \mathrm{LO} / \mathrm{I}_{1} \mathrm{LO}$ ratio in the hexagonal phase. The change of the electron-phonon coupling (as observed in the intensity of the LO ratio) is attributed to the change in the structure [23]. The electron-phonon coupling in bulk cubic CdS is found to be lower than the hexagonal CdS. It is due to the difference in the symmetries of LO phonon as well as that in the nature of the interband transition in the two phases. We observe that the values of $\mathrm{I}_{2} \mathrm{LO} / \mathrm{I}_{1} \mathrm{LO}$ for samples with mass composition of $3: 1$ onwards are higher. These values are measured with the suppression of the baseline, and therefore, they show precisely the actual values of $\mathrm{I}_{2} \mathrm{LO} / \mathrm{I}_{1} \mathrm{LO}$. The Raman curves in the current study are also shifted up as the mass composition of cadmium precursor increases. This phenomenon indicates that Raman fluorescence is present as the mass composition of the cadmium chloride increased. The correlation between the Raman spectrum and photoluminescence spectra shall be discussed further in the photoluminescence section later.

3.1.5. UV-Visible Spectroscopy. Figure 6 shows the UV absorption for samples at various mass compositions. The absorption peak of the bulk CdS was reported to be around $512 \mathrm{~nm}$ [26]. All the samples are blue shifted in comparison to the band gap value of the bulk CdS (512 nm) [26]. The blue shift of the optical absorption edge indicates the formation of particles in the nanometer size regime [6]. When the mass composition of the sample is at $1: 2$, the UV absorption 

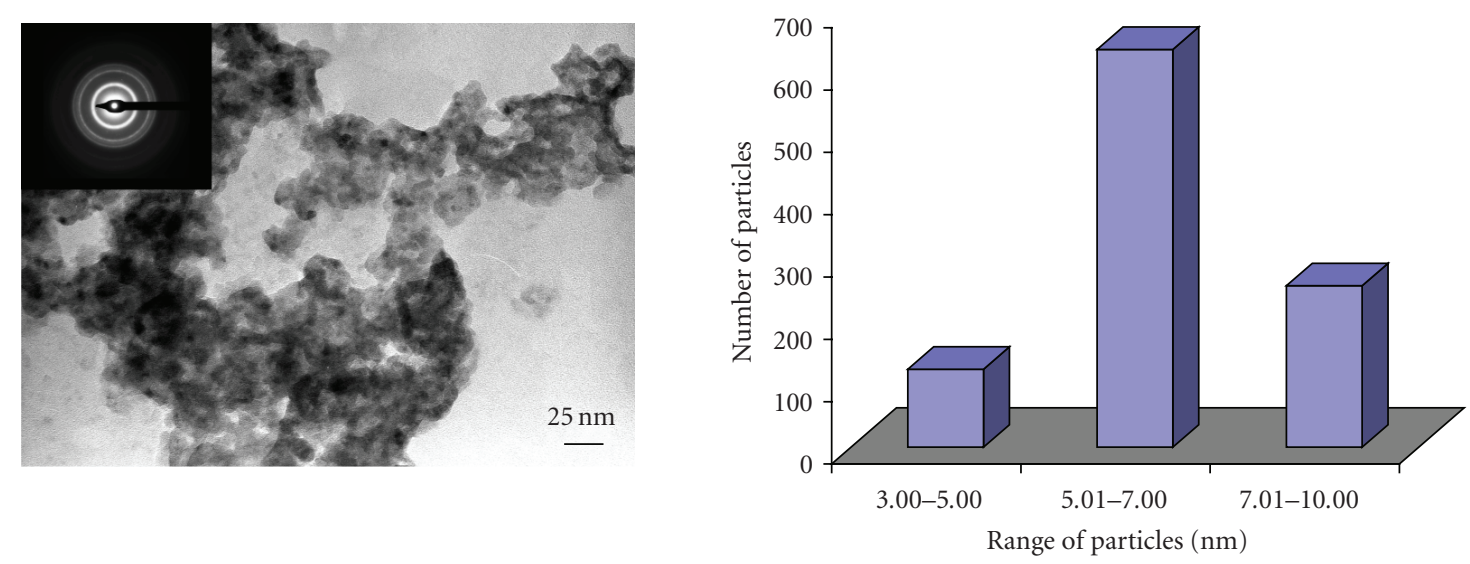

(a)
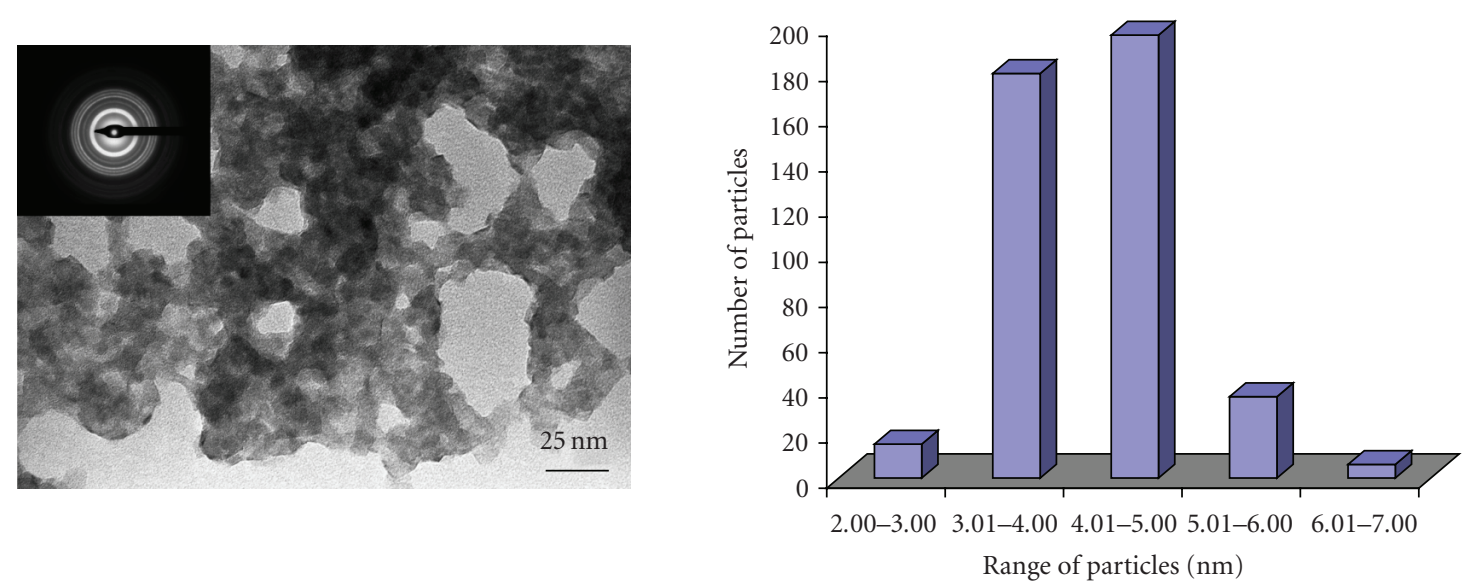

(b)
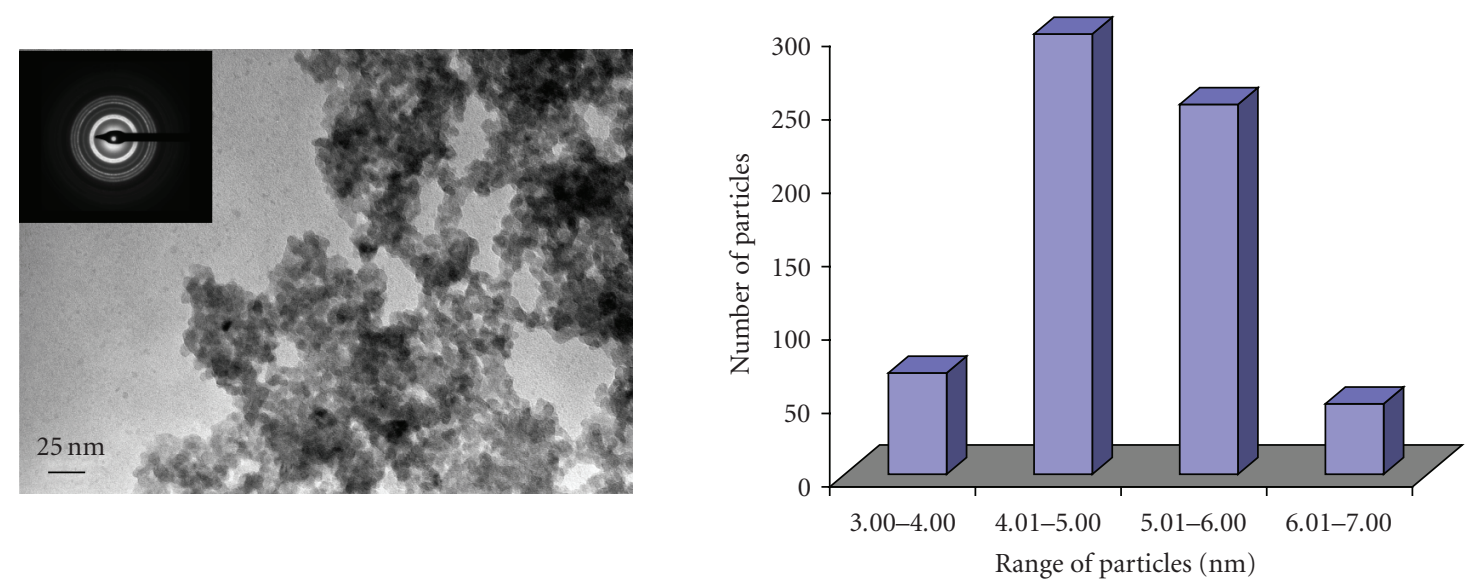

(c)

FIGURE 3: TEM microscope images and the histogram of the size distribution of the as-prepared CdS: (a) CdS (1:2), (b) CdS (3:1), and CdS (6:1) .Insert : SAED

shoulder is $475 \mathrm{~nm}$. However, when the mass composition is increased to $3: 1$ and $6: 1$, respectively, the absorption bands become broader and the intensity of the peaks also increases two fold. The UV absorption peaks for these samples at 3 : 1 and $6: 1$ are around $480 \mathrm{~nm}$ as illustrated in Figure 6. However, the absorption onsets for all samples are near to band edge of the bulk CdS.
3.1.6. X-Ray Photoelectron Spectroscopy (XPS). The chemical compositions of CdS at different cadmium to sulfur compositions at $1: 2,3: 1$ and $6: 1$ are determined with XPS measurements. The XPS is similar to the typical CdS spectrum reported by other researchers' work [27-29]. Figure 7 shows the presence of $\mathrm{Cd}$ and $\mathrm{S}$ elements in the samples. The chemical state of the nanocrystals has been 


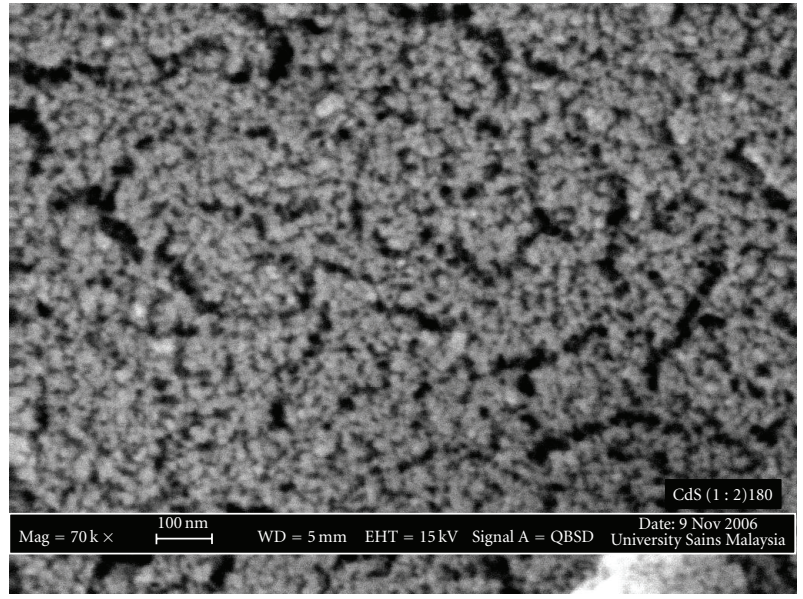

FIGURE 4: SEM images of as-prepared CdS nanoparticles.

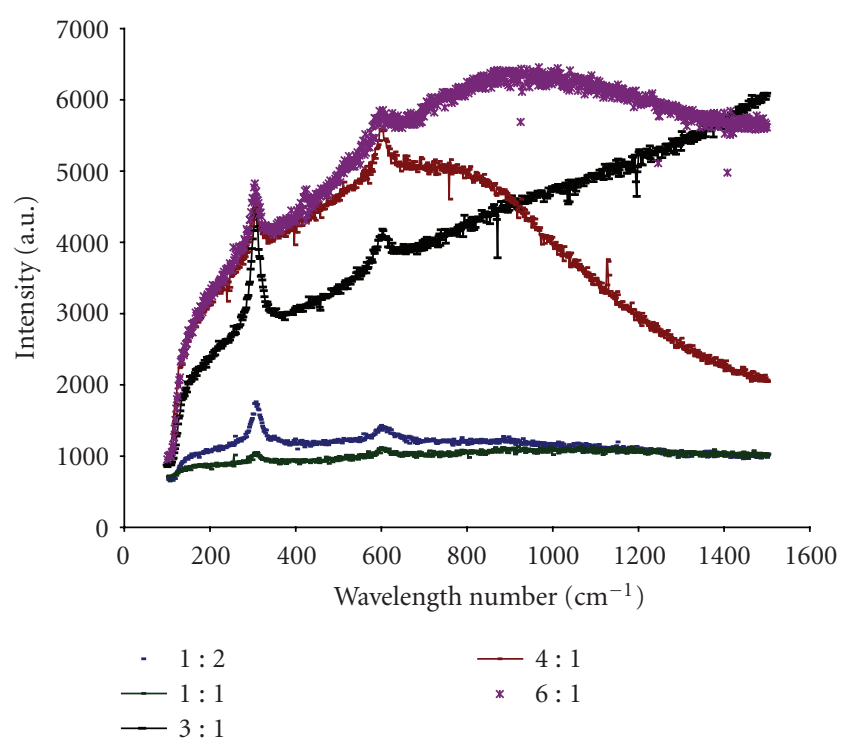

FIgURE 5: Raman spectroscopy for sample at mass composition at $1: 2,1: 1,3: 1,4: 1$, and $6: 1$ (cadmium chloride: thiourea) at $160^{\circ} \mathrm{C}$.

investigated by high-resolution XPS. Figure 7 shows the XPS spectra of the Cd3d core and S2p core, respectively. There are two states for sulfur which are $S 2 p_{1 / 2}$ and $S 2 p_{3 / 2}$ and the spin orbit splitting between these two states is $1.2 \mathrm{eV}$ [30]. In the sample of mass composition $1: 2$, two strong peaks of $\mathrm{Cd} 3 \mathrm{~d}$ are observed at 406.1 and $413.1 \mathrm{eV}$ whereas the binding energy of $\mathrm{S} 2 \mathrm{p}_{3 / 2}$ is $162.1 \mathrm{eV}$ which is consistent with the reported value of CdS [31].

The Cd3d spectrum has a doublet feature due to spinorbit splitting resulting into $3 \mathrm{~d}_{5 / 2}$ and $3 \mathrm{~d}_{3 / 2}$ peaks with spinorbit separation of $7.3 \mathrm{eV}$. In a sample with mass composition 3:1. two peaks of core $\mathrm{Cd} 3 \mathrm{~d}$ are observed at $406.38 \mathrm{eV}$ and $413.7 \mathrm{eV}$, respectively. A satisfactory is obtained using a single spin-orbit pair. Similarly, the binding energy for S2p is $162.5 \mathrm{eV}$ that agrees well with the reported value of CdS. Lastly, a sample with mass composition of $6: 1$ displays

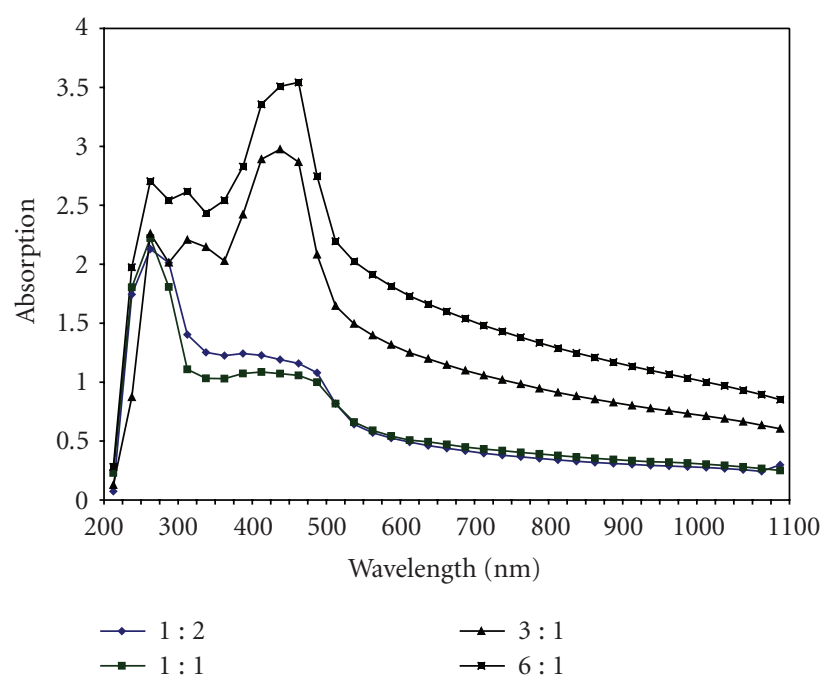

FIgURE 6: UV absorption at various mass composition at (a) $1: 2$ (b) $1: 1$ (c) $3: 1$, and (d) $6: 1$ (cadmium chloride : thiourea).

two strong peaks of $\mathrm{Cd} 3 \mathrm{~d}$ at $406.3 \mathrm{eV}$ and $413.2 \mathrm{eV}$ that correspond with $C d 3 d_{5 / 2}$ and $C d 3 d_{3 / 2}$. The binding energy for $\mathrm{S}_{2} \mathrm{p}_{3 / 2}$ is also $162.8 \mathrm{eV}$. The strong peaks $\mathrm{Cd} 3 \mathrm{~d}_{5 / 2}$ and S2 $\mathrm{p}_{3 / 2}$ have electron binding energies at around $406 \mathrm{eV}$ and 162-163 eV which suggest the valence states of $\mathrm{Cd}$ and $\mathrm{S}$ are +2 and -2 , respectively. Besides that, we also observe the state from $\mathrm{Cd}$ at around $404 \mathrm{eV}$. We can see also on these spectra the $\mathrm{Cd} 3 \mathrm{p}_{3 / 2}$ and $\mathrm{Cd} 3 \mathrm{p}_{1 / 2}$ level at 620 and $654 \mathrm{eV}$ and two smaller peaks around $10 \%$ of the main peaks shifted by $10 \mathrm{eV}$ toward lower energies. These two small peaks are due to by the monochromatocity of our X-ray source.

These are two $\mathrm{Cd} 3 \mathrm{~d}_{5 / 2}$ peaks, namely, $\mathrm{Cd} 3 \mathrm{~d}_{5 / 2} \mathrm{CdS}$ and $\mathrm{Cd} 3 \mathrm{~d}_{5 / 2}$ CdClx. Peak Cd3d $\mathrm{d}_{5 / 2} \mathrm{CdClx}$ is attributed to the under stochiometry that occurs at around $405 \mathrm{eV}$ depending on the charge effect during the analysis. When we plot the area under the curve of $\mathrm{Cd} 3 \mathrm{~d}_{5 / 2}$ CdClx over the area under the curve of $\mathrm{Cd} 3 \mathrm{~d}_{5 / 2} \mathrm{CdS}$, we find that ratio slightly increases as the concentration of CdS in the samples increases. Therefore, it indicates that the under stoichiometry or sulfur vacancy occurs as the concentration of CdS increases in the samples. The measurement of sulfur vacancy could also be represented in the ratio of integral area for Cd3d to S2p as described by Pan and his colleagues [32]. Hence, the ratios of our three samples at different mass compositions $(1: 2,3$ $: 1$ and $6: 1\left(\mathrm{CdCl}_{2}:\right.$ thiourea, $\left.\left.\mathrm{w} / \mathrm{w}\right)\right)$ are $1.2: 1.0,1.1: 1.0$, and $1.4: 1.0$, respectively. These values support that sulfur vacancies take place in our samples. Pan and his colleagues have reported that the ratio of integral area for $\mathrm{Cd} 3 \mathrm{~d}$ to S2p in their work was $1.03: 1.00$ and they suggested that the surface defects such as sulfur vacancies and/or sulfur dangling bonds occurred in the sample.

In the present study, the analyzed samples are considered to be homogeneous although there is a slight presence of $\mathrm{Cl}^{-}$ ions that originates from the residue of cadmium chloride during the reaction. We could also detect oxygen peaks at binding energies of $532.4 \mathrm{eV}, 533.1 \mathrm{eV}$, and $533.1 \mathrm{eV}$ in samples of different mass compositions of $1: 2,3: 1$, and $6: 1$, 


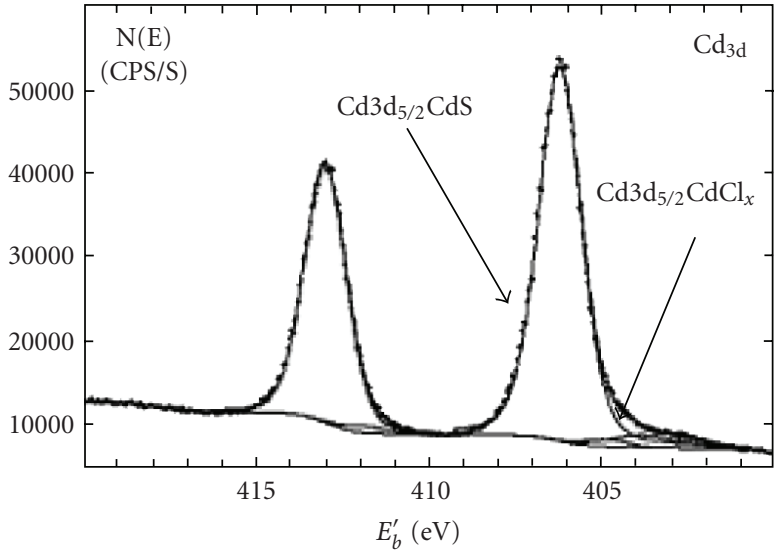

(a)

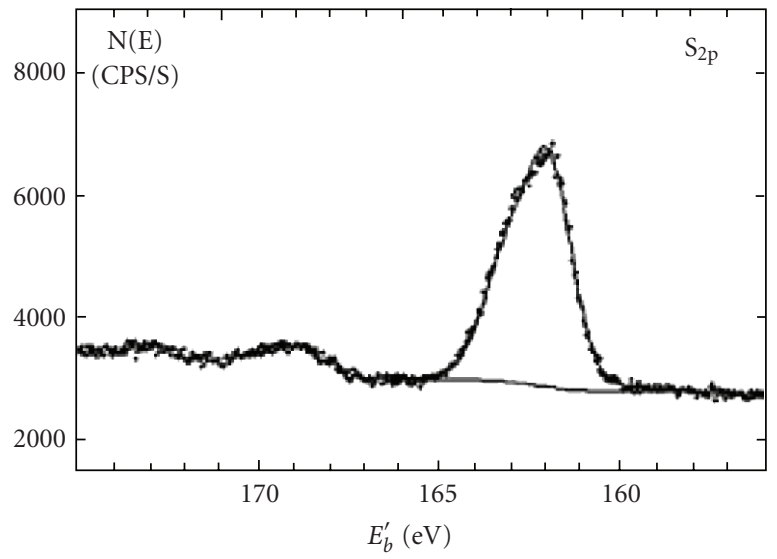

(b)

FIgURE 7: XPS spectra of CdS nanoparticles at mass composition $1: 2$.

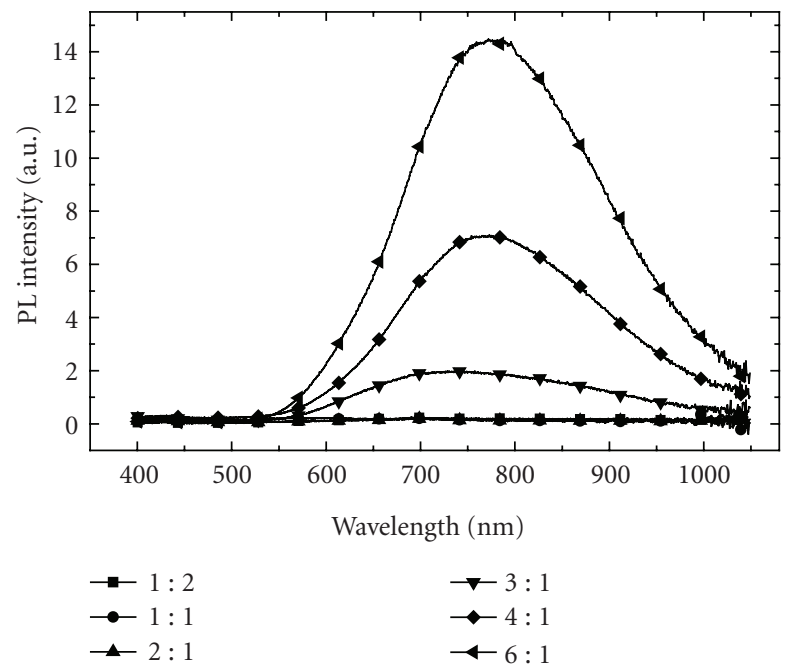

FIGURE 8: PL spectra of CdS nanoparticles at different mass compositions of cadmium chloride and thiourea at room temperature.

respectively. The $\mathrm{O}$ peak could be attributed to the presence of $\mathrm{H}_{2} \mathrm{O}$ from atmosphere adsorbed on the sample surface. The result is consistent with the Raman spectroscopy finding in which the Raman fluorescence are observed for ratio $3: 1$ and $6: 1$ (Figure 3).

3.1.7. Photoluminescence ( $P L)$ Emission. Figure 8 shows the room temperature PL spectra of samples CdS (1:2) to CdS (6 : 1). The PL spectra show a broad band with a maximum around $760 \mathrm{~nm}$. For a Cd concentration lower or equal to that corresponding to a $\mathrm{Cd} / \mathrm{S}$ mass composition equal to 2 : 1 , no PL is observed. For higher Cd content, the PL intensity is an increasing function of the Cd content. The observation correlates with the Raman results which show the fluorescence effect from sample $3: 1$ to $6: 1$. We could observe that the intensity of PL increases when the mass composition of $\mathrm{Cd}^{2+}$ ions is $3: 1$ onwards as illustrated in Figure 8. Hence, the presence of fluorescence could be the indicator of the PL existence in the samples. The low energy of this PL band shows that the PL is not due to the excitonic emission of CdS. Moreover the excitonic PL band is generally observed around $410 \mathrm{~nm}$ for confined CdS nanoparticles [33]. As the PL peak energies are less than the band gap energy, the luminescence bands can be identified with transitions involving donors, acceptors, and surface traps [34]. This band is most likely caused by the recombination through deep defect levels [35] when compared with the bulk CdS $(512 \mathrm{~nm})$. It also suggests that this low-energy band could originate from donor-acceptor recombination involving deep state in the CdS bandgap [36]. Therefore, the defect states contributing to the low-energy band edge PL are highly attributed to the native defects such as sulfur or cadmium vacancies [36]. In the present study, the low-energy band is probably due to the sulfur vacancies.

Bare CdS nanocrystals usually exhibit low photoluminescence (PL) intensity attributed to their surface states arising from the surface nonstoichiometry, unsaturated bonds and so forth. Besides that, they are also not stable when exposed to air and light due to the photooxidation at the surface [3]. It was reported that the CdS nanoparticles often present a broad low-energy luminescence attributed to the midgap surface states with a very weak intensity [37]. In the current study, we observe that PL intensity from CdS $(1: 2)$ till CdS $(2: 1)$ is very low. The origin that accounts for the weaker PL emission of these samples could be attributed to a lack of sulfur vacancy complexes as most of the photoexcited electrons in CdS cannot be transferred into the luminescence centers and relax radiatively. Thus, the weak PL emission indicates a lack of luminescence centers. However, as the ratio of $\mathrm{Cd}^{2+}$ is increased, the amount of sulfur vacancies are also increased which could explain that the luminescence intensity is also strongly enhanced [38]. $\mathrm{S}^{2-}$ vacancies can act as a deep trap for photogenerated electrons in the conduction band, resulting in the red luminescence of CdS through transfer to surface molecules or to pre-existing trapped holes [4]. Nonband edge photoluminescence is observed and this 


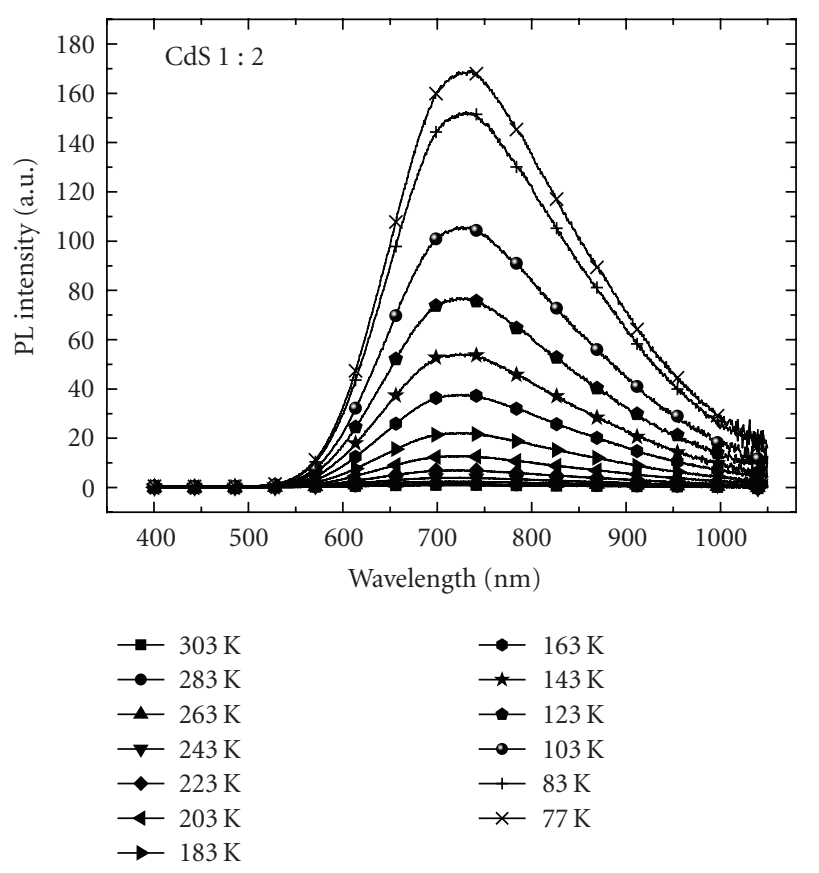

FIgURE 9: Temperature dependence of photoluminescence for CdS nanoparticles at mass composition $1: 2$.

could be due to the fact that the CdS nanoparticles are in contact with each other as observed in the TEM images earlier. Quantum confinement is not observed, most likely due to the aggregation of the nanoparticles.

Subsequently, the temperature dependence of photoluminescence is studied in the range $77-303 \mathrm{~K}$. The spectra of the temperature are shown in Figure 9. Similar studies on temperature dependence of photoluminescence have been carried out by several researchers [39-41]. Whereas the sample did not present any measurable PL at room temperature, the photoluminescence intensity increased as the temperature decreased. There is no significant shift as the temperature is decreasing. The shape of the PL band remains unchanged. However, the intensity of the photoluminescence increases 183 -fold when the temperature is at $77 \mathrm{~K}$. This evolution strongly suggests that thermally activated nonradiative mechanisms exist, which quench the $\mathrm{PL}$ at room temperature.

The obtained results indicate that CdS nanoparticles are promising for PL at higher energy with the condition that we could disperse the nanoparticles well. Densely packed CdS nanoparticles would behave as bulk CdS. Furthermore, the presence of surface states and/or vacancies has proven to involve in trap-related luminescence.

\section{Conclusions}

We have successfully synthesized bare CdS based on thermolysis of a single-source organometallic precursor. The origin of $\mathrm{Cd}^{2+}$ source has been shown to influence the formation of the crystalline phase. In this case, manipulation of mass composition of $\mathrm{CdCl}_{2}$ is found to assist in transforming the crystalline phase from cubic to hexagonal. TEM result reveals that the CdS nanoparticles structures are irregular spherical shapes and are in contact with each other. The average particle size in all samples acquired is less than $7 \mathrm{~nm}$ with the standard deviation being less than $1 \mathrm{~nm}$. XPS result indicates the presence of sulfur vacancies in the samples. In the PL studies, the results demonstrate that the intensity increases as the mass composition of cadmium chloride increases and all their emissions fall in the range of $700-800 \mathrm{~nm}$. We conclude that the presence of sulfur vacancies and the fact that bare CdS nanoparticles are aggregated have contributed significantly towards the red-emission in PL spectra. It would be interesting to perform a similar study on the molecularcapped CdS nanoparticles which would further justify our current conclusion.

\section{Acknowledgments}

The authors would like to acknowledge the contribution by Professor Patrice Bourson from Laboratoire Matériaux Optiques Photoniques et Systemes in the University of Metz for Raman Spectroscopy analysis. This research is supported in part by Malaysia Toray Scientific Foundation (MTSF) Science and Technology Grant 2005 (304/PKimia/650343/T102) and USM Short Term Grant (304/PKimia/638035). The authors also wish to thank ASTS, USM and the French Government for the financial support in this project.

\section{References}

[1] T. Di Luccio, E. Piscopiello, A. M. Laera, and M. V. Antisari, "Structural studies of thin films of semiconducting nanoparticles in polymer matrices," Materials Science and Engineering C, vol. 27, no. 5-8, pp. 1372-1376, 2007.

[2] A. P. Alivisatos, "Semiconductor clusters, nanocrystals, and quantum dots," Science, vol. 271, no. 5251, pp. 933-937, 1996.

[3] L. Xu, L. Wang, X. Huang, J. Zhu, H. Chen, and K. Chen, "Surface passivation and enhanced quantum-size effect and photo stability of coated CdSe/CdS nanocrystals," Physica E, vol. 8, no. 2, pp. 129-133, 2000.

[4] S. Celebi, A. K. Erdamar, A. Sennaroglu, A. Kurt, and H. Y. Acar, "Synthesis and characterization of poly(acrylic acid) stabilized cadmium sulfide quantum dots," Journal of Physical Chemistry B, vol. 111, no. 44, pp. 12668-12675, 2007.

[5] R. Banerjee, R. Jayakrishnan, and P. Ayyub, "Effect of the sizeinduced structural transformation on the band gap in CdS nanoparticles," Journal of Physics: Condensed Matter, vol. 12, no. 50, pp. 10647-10654, 2000.

[6] M. Pattabi and B. S. Amma, "Synthesis and stability studies of thiophenol capped CdS nanoparticles," Solar Energy Materials and Solar Cells, vol. 90, no. 15, pp. 2377-2383, 2006.

[7] V. Singh, B. P. Singh, T. P. Sharma, and R. C. Tyagi, "Effect of ambient hydrogen sulphide on the optical properties of evaporated cadmium sulphide films," Optical Materials, vol. 20, no. 3, pp. 171-175, 2002.

[8] Y.-J. Zhu and H. Tong, "Synthesis of CdS nanocrystals based on low-temperature thermolysis of one single-source organometallic precursor," Nanotechnology, vol. 17, no. 3, pp. 845-851, 2006. 
[9] M. Gao, X. Zhang, B. Yang, F. Li, and J. Shen, "Assembly of modified CdS particles/cationic polymer based on electrostatic interactions," Thin Solid Films, vol. 284-285, pp. 242245, 1996.

[10] J. Kim, S. S. Kim, K. H. Kim, et al., "Applications of telechelic polymers as compatibilizers and stabilizers in polymer blends and inorganic/organic nanohybrids," Polymer, vol. 45, no. 10, pp. 3527-3533, 2004.

[11] C.-H. Fischer and E. Kenndler, "Analysis of colloids: IX. Investigation of the electrical double layer of colloidal inorganic nanometer-particles by size-exclusion chromatography," Journal of Chromatography A, vol. 773, no. 1-2, pp. 179-187, 1997.

[12] J. R. L. Fernandez, M. de Souza-Parise, and P. C. Morais, "Optical investigation of the red band emission of CdS nanoparticles," Surface Science, vol. 601, no. 18, pp. 38053808, 2007.

[13] J. Zhao, K. Dou, Y. Chen, et al., "Temperature dependence of photoluminescence in CdS nanocrystals prepared by the sol-gel method," Journal of Luminescence, vol. 66-67, no. 1-6, pp. 332-336, 1995.

[14] M. Ichimura, F. Goto, and E. Arai, "Structural and optical characterization of CdS films grown by photochemical deposition," Journal of Applied Physics, vol. 85, no. 10, pp. 7411-7417, 1999.

[15] L. A. Patil, P. A. Wani, and D. P. Amalnerkar, " $\mathrm{CuCl}_{2}$ assisted cubic to hexagonal phase transformation of cadmium sulphide," Materials Chemistry and Physics, vol. 61, no. 3, pp. 260-265, 1999.

[16] K. Murakoshi, H. Hosokawa, M. Saitoh, et al., "Preparation of size-controlled hexagonal CdS nanocrystallites and the characteristics of their surface structures," Journal of the Chemical Society, Faraday Transactions, vol. 94, no. 4, pp. 579-586, 1998.

[17] X. L. Tong, D. S. Jiang, Z. M. Liu, et al., "Structural characterization of CdS thin film on quartz formed by femtosecond pulsed laser deposition at high temperature," Thin Solid Films, vol. 516, no. 8, pp. 2003-2008, 2008.

[18] A. J. Wagner, G. M. Wolfe, and D. H. Fairbrother, "Reactivity of vapor-deposited metal atoms with nitrogen-containing polymers and organic surfaces studied by in situ XPS," Applied Surface Science, vol. 219, no. 3-4, pp. 317-328, 2003.

[19] S. Prabahar and M. Dhanam, "CdS thin films from two different chemical baths_-structural and optical analysis," Journal of Crystal Growth, vol. 285, no. 1-2, pp. 41-48, 2005.

[20] M. Z. Rong, M. Q. Zhang, H. C. Liang, and H. M. Zeng, "Surface modification and particles size distribution control in nano-CdS/polystyrene composite film," Chemical Physics, vol. 286, no. 2-3, pp. 267-276, 2003.

[21] K. Matsumoto, K. Takagi, and S. Kaneko, "Kinetics of the cubic $\rightarrow$ hexagonal transformation of cadmium sulfide," Journal of the Electrochemical Society, vol. 130, no. 2, pp. 423-426, 1983.

[22] M. Abdulkhadar and B. Thomas, "Study of raman spectra of nanoparticles of CdS and ZnS," Nanostructured Materials, vol. 5, no. 3, pp. 289-298, 1995.

[23] V. Sivasubramanian, A. K. Arora, M. Premila, C. S. Sundar, and V. S. Sastry, "Optical properties of CdS nanoparticles upon annealing," Physica E, vol. 31, no. 1, pp. 93-98, 2006.

[24] O. Zelaya-Angel, F. D. L. Castillo-Alvarado, J. AvendañoLópez, et al., "Raman studies in CdS thin films in the evolution from cubic to hexagonal phase," Solid State Communications, vol. 104, no. 3, pp. 161-166, 1997.

[25] J. J. Shiang, S. H. Risbud, and A. P. Alivisatos, "Resonance Raman studies of the ground and lowest electronic excited state in CdS nanocrystals," The Journal of Chemical Physics, vol. 98, no. 11, pp. 8432-8442, 1993.
[26] L. Xu, Y. Su, D. Cai, Y. Chen, and Y. Feng, "Synthesis and photoluminescence properties of CdS nanobelts," Materials Letters, vol. 60, no. 11, pp. 1420-1424, 2006.

[27] L. Mirenghi, F. Antolini, and L. Tapfer, "Influence of the matrix on the chemical surface bonds of $\mathrm{ZnS}$ and $\mathrm{CdS}$ nanoclusters obtained by chemical synthesis," Surface and Interface Analysis, vol. 38, no. 4, pp. 462-468, 2006.

[28] P. S. Khiew, S. Radiman, N. M. Huang, and M. Soot Ahmad, "Studies on the growth and characterization of CdS and $\mathrm{PbS}$ nanoparticles using sugar-ester nonionic water-in-oil microemulsion," Journal of Crystal Growth, vol. 254, no. 1-2, pp. 235-243, 2003.

[29] M. Takahashi, S. Hasegawa, M. Watanabe, T. Miyuki, S. Ikeda, and K. Iida, "Preparation of CdS thin films by electrodeposition: effect of colloidal sulfur particle stability on film composition," Journal of Applied Electrochemistry, vol. 32, no. 4, pp. 359-367, 2002.

[30] R. S. C. Smart, J. Amarantidis, W. M. Skinner, C. A. Prestidse, L. L. Venier, and S. R. Grano, "Solid-liquid interfaces: macroscopic phenomena-microscopic understanding adsorption," in Processing Techniques, K. Wandelt and S. Thurgate, Eds., p. 42, Springer, New York, NY, USA, 2003.

[31] G. Hota, S. B. Idage, and K. C. Khilar, "Characterization of nano-sized CdS-Ag2S core-shell nanoparticles using XPS technique," Colloids and Surfaces A, vol. 293, no. 1-3, pp. 5-12, 2007.

[32] Q. Pan, K. Huang, S. Ni, Q. Wang, F. Yang, and D. He, "Fabrication and photoluminescence properties of large-scale hierarchical CdS dendrites," Materials Letters, vol. 61, no. 26, pp. 4773-4776, 2007.

[33] P. K. Khanna and N. Singh, "Light emitting CdS quantum dots in PMMA: synthesis and optical studies," Journal of Luminescence, vol. 127, no. 2, pp. 474-482, 2007.

[34] K. K. Nanda, S. N. Sarangi, and S. N. Sahu, "Visible light emission from CdS nanocrystals," Journal of Physics D, vol. 32, no. 17, pp. 2306-2310, 1999.

[35] D. Nesheva, C. Raptis, Z. Levi, Z. Popovic, and I. Hinic, "Photoluminescence of CdSe nanocrystals embedded in a $\mathrm{SiO}_{x}$ thin film matrix," Journal of Luminescence, vol. 82, no. 3, pp. 233-240, 1999.

[36] A. Chahboun, A. G. Rolo, S. A. Filonovich, and M. J. M. Gomes, "Factors influencing the passivation of CdS quantum dots embedded in silica glass," Solar Energy Materials and Solar Cells, vol. 90, no. 10, pp. 1413-1419, 2006.

[37] Y. Li, F. Huang, Q. Zhang, and Z. Gu, "Solvothermal synthesis of nanocrystalline cadmium sulfide," Journal of Materials Science, vol. 35, no. 23, pp. 5933-5937, 2000.

[38] F. Chen and W. Jie, "Growth and photoluminescence properties of CdS solid solution semiconductor," Crystal Research and Technology, vol. 42, no. 11, pp. 1082-1086, 2007.

[39] E. A. Bagaev, K. S. Zhuravlev, and L. L. Sveshnikova, "Temperature dependence of photoluminescence of CdS nanoclusters formed in the Langmuir-Blodgett film matrix," Semiconductors, vol. 40, no. 10, pp. 1188-1192, 2006.

[40] S. Santhi, E. Bernstein, and F. Paille, "Temperature dependence of trap luminescence of CdS doped glasses," Journal of Luminescence, vol. 117, no. 1, pp. 101-112, 2006.

[41] A. A. Bol, R. van Beek, J. Ferwerda, and A. Meijerink, "Temperature dependence of the luminescence of nanocrystalline CdS/Mn ${ }^{2+}$," Journal of Physics and Chemistry of Solids, vol. 64, no. 2, pp. 247-252, 2003. 

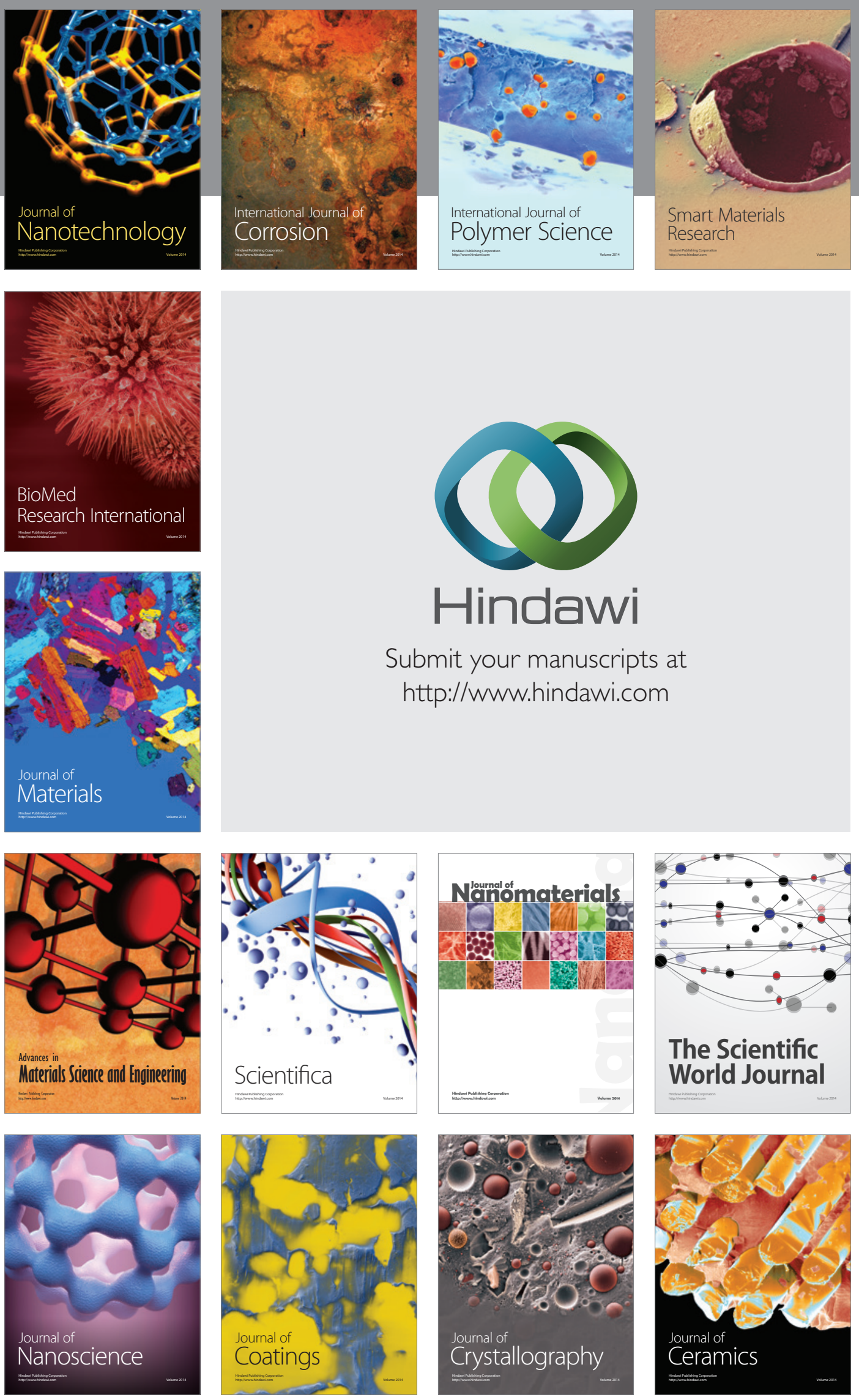

The Scientific World Journal

Submit your manuscripts at

http://www.hindawi.com

\section{World Journal}

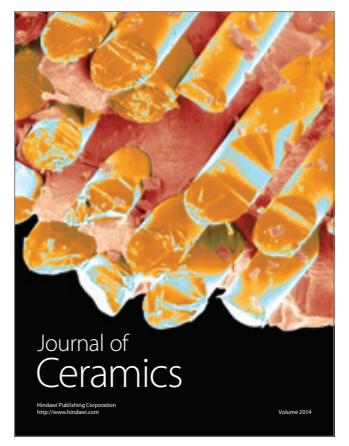

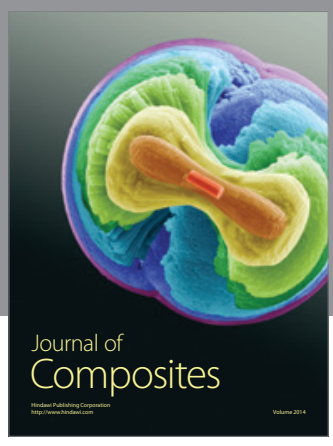
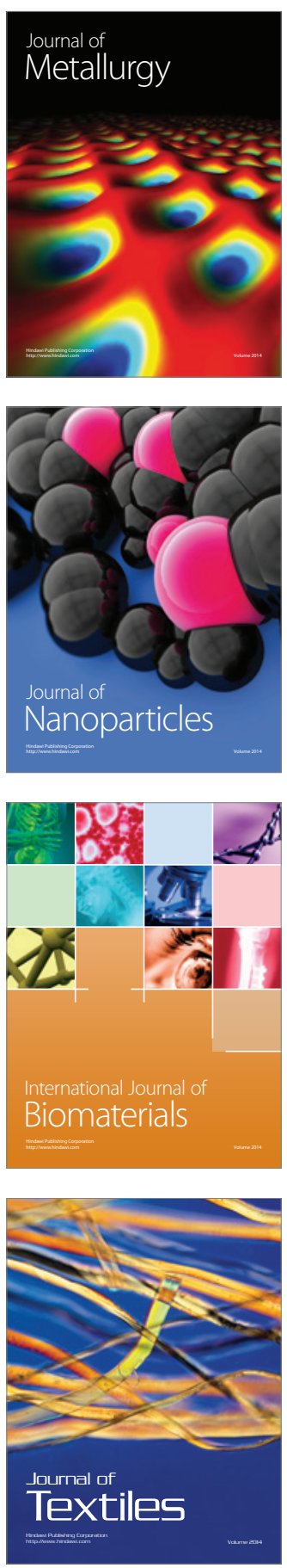